\title{
UN PROGETTO PER IL RILIEVO ARCHEOLOGICO DEL SITO DI MASADA IN ISRAELE
}

\author{
Nota del m.s. SANDRO PARRINELLO (*)
}

(Adunanza del 7 maggio 2015)

SunTO. - Il testo propone una riflessione sul disegno ed il rilievo architettonico come strumento per lo studio dei reperti archeologici. La descrizione del sito di Masada e delle vicende che ne hanno caratterizzato la storia più recente fino alla definizione delle strategie documentali messe a sistema per lo sviluppo di un percorso di conoscenza condotto a cavallo tra didattica e ricerca.

$* * *$

ABSTRACT. - The text proposes a reflection on drawing and architectural survey as a tool for the study of archaeological sites. The description of the Masada site and the events that have characterized its most recent history till to the definition of the documentary strategies have established a system for the development of a path of knowledge developed in synergy between didactic and research.

\section{Premessa SUl RILIEVO ARCHEOLOGICO}

Lo studioso dell'architettura è senza dubbio attratto dallo studio dell'antico. Questa ricerca è sempre stata svolta sia con l'ausilio di fonti storiche e letterarie, sia mediante la conoscenza diretta dei resti della produzione architettonica del passato, mediante il disegno e la rilevazione. Come dimostrano gli studi degli umanisti rinascimentali del quindicesimo secolo, artisti e architetti documentavano con schizzi, misurazioni e

(") DICAr - Dipartimento di Ingegneria Civile e Architettura, Università di Pavia, Italy. E-mail: sandro.parrinello@unipv.it 
rappresentazioni di vario tipo, oltre che con accurate descrizioni letterarie, le vestigia del passato. Fino al XVII secolo sono tuttavia le grandi problematiche legate all'architettura e non all'archeologia ad essere oggetto delle indagini dei trattatisti i quali, pur interessandosi dell'architettura antica, elaborano teorie e metodiche suffragate in parte dal rilevamento dei resti degli antichi edifici. L'attività del rilievo appare spesso come una esercitazione scolastica, fondamentale per la formazione degli architetti, ma raramente queste esperienze assumono una valenza di lavoro autonomo finalizzato allo studio archeologico. Verso la fine del XVII secolo si sviluppano esigenze di più accurati studi sull'architettura antica e si avvisa la necessità di avere a disposizione analisi più attente, basate su rilievi dettagliati dei resti dell'antichità: il rilievo architettonico, quale strumento di conoscenza e divulgazione culturale, diviene oggetto di numerose opere a stampa anche a carattere divulgativo. Nel XVIII secolo la scoperta e l'esplorazione delle città sepolte dall'eruzione del Vesuvio di Pompei ed Ercolano (Italia) contribuirono allo sviluppo di una ricerca più sistematica dei resti antichi, portata avanti in seguito, su basi più o meno scientifiche, fino alle scoperte di Schliemann che operò negli anni 18701880 a Troia (Turchia) e Micene (Grecia).

Nei primissimi anni dell' Ottocento vennero realizzate importanti esperienze dai rilevatori al seguito delle campagne napoleoniche; le restituzioni di Vivant Dénon delle vestigia egizie sono un esempio di fedeltà all'oggetto, con una accurata considerazione dello stato di conservazione degli edifici fino alla rappresentazione delle lesioni delle singole pietre. ${ }^{1}$ Nel 1812 l'esploratore svizzero Johann Ludwig Burkhardt ${ }^{2}$ rivelò al mondo occidentale le splendide architetture rupestri di Petra, conservatesi per la maggior parte quasi intatte sia per le caratteristiche geomorfologiche del sito sia per la singolare tecnica di realizzazione monolitica. Il grande successo dei primi resoconti, arricchiti da ottime illustrazioni grafiche $e^{3}$ e, soprattutto, da una serie di splendide litografie realizzate da David Roberts ${ }^{4}$ edite fra il 1842 ed il 1849 (tratte da dise-

1 D.V. Denon, Voyage dans la Basse et la Haute Egypte, ed. It. Firenze, 1808.

2 J.L. Burkhardt, Travels in Syria and the Holy Land, London, 1822, pp. 420-434

3 L.M. Leonde, Journey through Arabia Petrea to Mount Sinai and the axcavated city of Petra the Edom of the Prophecies, $2^{\text {nd }}$ ed. , London, 1838. C.f.r. anche L. Marino, I disegnatori di Petra, in AA. VV. Il disegno luogo della memoria, atti del convegno di Firenze, 21-23 settembre 1995, Firenze, Alinea, 1995.

4 Raccolta di vedute di D. Roberts, Londra, 1839, cfr. D. Roberts, Journey to 
gni realizzati nel 1839 durante una apposita spedizione dall'Egitto alla Terrasanta), contribuì ad incrementare l'interesse per la mitica città.

Il XIX secolo è caratterizzato anche dallo sviluppo della attività dei pensionnaires accreditati presso l'Accademia di Francia a Roma. ${ }^{5} \mathrm{La}$ caratteristica di questi studi consiste nel rilievo del rudere e nella successiva operazione di "restauro", ovvero di ricostruzione del monumento a livello grafico, basata sui reperti dell'eventuale scavo e soprattutto sui dettami delle teorie degli ordini classici.

Tra il 1895 ed i primissimi anni del Novecento, con la scoperta del mondo preclassico, architetti - archeologi dalle specifiche competenze operano nei cantieri più importanti quali Susa, Babilonia, Assur e Bogazkoy. Con Evans, autore della eccezionale scoperta del mondo minoico (1900-1905), operano vari architetti tra i quali Théodore Fyfe, Christian Doll e Piet de Jong, che contribuiscono alla redazione dei rilievi e delle suggestive rappresentazioni grafiche oltre che alle audaci ricostruzioni in situ. ${ }^{6}$

Il disegno dell'antico e la creazione di strumenti rappresentativi attraverso i quali apprendere le ragioni compositive dell'architettura storica, hanno portato nel corso dell'ultimo secolo ha sviluppare numerose metodologie di rappresentazione grafica per la trasposizione del sistema reale rappresentato, in disegno da assimilare per consentire poi l'espressione dell'idea che sottende il progetto.'

Nel quadro di un generale contesto che tende oggi a rivalutare la specificità della figura e delle competenze del rilevatore per gli aspetti della conservazione e del restauro, si assiste ad un progressivo crescente interesse per questa figura professionale nel campo delle indagini archeologiche che, nell'ambito specifico, tende ad assumere un ruolo rilevante ed a ritagliarsi un autonomo spazio di ricerca.

Petra end the Holy Land, introduzione e commenti di E. Nistri, trad. inglese di Paula Boomsliter, Firenze, 1997.

5 M. Docci, D. Maestri, Storia del rilevamento architettonico e urbano, Laterza, Bari, 1993, p. 221.

6 D. Laroche, Alla scoperta dell'antichità preclassica, in "Rassegna" ("L’Archeologia degli architetti”), XV, n. 55/3, 1993, pp. 68-73.

7 Per una trattatistica generale sullo sviluppo della disciplina del rilievo architettonico Cfr. M. Docci, D. Maestri, Storia del rilevamento architettonico e urbano, Cit.; Cfr. anche S. Bertocci, M. Bini, Manuale di rilievo architettonico e urbano, Città Studi Edizioni, Novara, 2012. 
L'archeologia è tradizionalmente suddivisa in discipline a seconda del periodo o della cultura oggetto di studio, oppure a seconda di particolari tecniche di indagine, di specifiche problematiche o ancora utilizza definizioni che si basano sulle tipologie dei materiali oggetto di studio. Si descrive quindi la disciplina in rapporto a ben precise specificità di settore che, in ogni caso, trattano del recupero sistematico e dello studio di reperti, 'antichità' in generale, e dunque di manufatti visti nel loro contesto. Il termine 'contesto' ha oggi implicazioni culturali, cronologiche, ma anche spaziali ed ambientali, pertanto l'architetto, per una propria specifica competenza ed attitudine a descrivere $\mathrm{i}$ fenomeni in termini di spazio e ambiente, fa parte a buon diritto degli studiosi che, nello specifico campo di ciascun progetto di indagine archeologica, hanno un ruolo fondamentale al pari delle altre specifiche figure professionali come gli addetti allo scavo ed i responsabili della registrazione dei reperti, operando assieme alle numerose figure di specialisti archeologi, storici, restauratori e coloro che si occupano delle scienze di laboratorio. ${ }^{8}$

Con lo sviluppo delle metodologie di indagine stratigrafica e dei metodi di ricerca correlati, che hanno comportato in genere una maggiore attenzione alle tracce della cultura materiale, ${ }^{9}$ si è giunti ad applicare le stesse metodologie di indagine allo studio delle strutture in elevato e quindi all'architettura in genere e non soltanto ai reperti allo stato di rudere: i campi di applicazione delle indagini architettoniche, strutturali, tipologiche, geometriche e formali, tipiche degli studi architettonici, hanno trovato quindi fertili e proficue applicazioni in archeologia.

Rilevare la struttura muraria ed i suoi corredi funzionali ed estetici, costituisce un'operazione che se condotta correttamente e finalizzata al confronto con strutture murarie, tessiture dei paramenti, caratteristi-

8 Si intendono, ad esempio, gli specialisti della datazione radiometrica o dell'identificazione dei pollini o della microfauna.

9 La principale tecnica di indagine archeologica è quella dello scavo stratigrafico che consente di rimuovere strati di terreno che si sono depositati nel bacino di un sito, generalmente antropizzato, rispettandone la relativa successione cronologica, e di documentare i materiali che vi sono rinvenuti, collocandoli in una sequenza cronologica relativa. L'indagine archeologica utilizza anche il confronto formale e stilistico con altri oggetti simili (ad esempio reperti fuori contesto), l'insieme delle tecniche scientifiche archeometriche, e può usufruire di tecniche di rilevamento e di datazione o di analisi scientifiche, come sopra esposto, elaborate da altre discipline. 
che costruttive e di finitura presenti nello stesso ambito territoriale, diviene per lo studio storico ed architettonico, oltre che sotto il profilo archeologico della cultura materiale, una condizione essenziale per la lettura delle strutture, utile soprattutto nel corso di analisi che investano siti di grande interesse ed estensione che presentano, come il caso di Masada in Israele, resti di edilizia a carattere monumentale sia tracce di edilizia minore o diffusa nel territorio.

Le tecnologie infografiche ed i sistemi di rilevamento digitale hanno inoltre trovato fertili applicazioni in vari campi delle indagini archeologiche $\mathrm{e}$, in primo luogo, offrono una quantità di applicazioni relative alle problematiche inerenti la gestione della vasta documentazione di scavo e di rilievo che ogni campagna produce. Uno dei principali obiettivi è in generale quello di costruire basi di dati, relative alla documentazione dei siti, facilmente consultabili ed aggiornabili, costruite in maniera da offrire la possibilità di consultazione e di gestione, anche on line, per gruppi di ricerca che, proprio per il carattere di interdisciplinarità delle ricerche, possono svilupparsi anche a livello internazionale. Questi risultano indispensabili strumenti per la gestione e la conservazione della documentazione di rilievo, sia degli appunti presi sul campo, sia dei rilievi realizzati con strumentazioni digitali, dai file sorgente agli elaborati intermedi e finali, delle immagini e delle foto, e possono offrire, se ben congegnati, la possibilità di effettuare ricerche ed indagini finalizzate alle esigenze didattiche o di studio di vari gruppi di interesse. L'adozione di tali strumenti, adattati alle esigenze dello specifico campo di indagine, consente, in prospettiva, la valorizzazione del cospicuo patrimonio iconografico e documentario che viene prodotto dal team di studiosi che operano in un determinato sito.

Elemento fortemente caratterizzante è infine la previsione della georeferenziazione G.P.S. dei dati topografici relativi ai soggetti trattati dagli elaborati grafici, che consente un rapido aggiornamento delle basi cartografiche con eventuali nuove acquisizioni (campagne di scavo). Le mappe digitali possono costituire la base di raccolta di sistemi di dati G.I.S. utili ad incrementare le possibilità di indagini che partano direttamente dalla cartografia di riferimento del sito e possano prevedere la restituzione di carte tematiche relative ai vari livelli di indagine. ${ }^{10}$ Una

10 Sono note le potenzialità offerte dai sistemi S.I.T o G.I.S. nel campo dell'archeologia in generale e, nello specifico, nel settore del rilievo archeologico e nei campi 
banca dati digitale dovrebbe costituire quindi la necessaria base informativa per la gestione di un'area archeologica, soprattutto in funzione della programmazione degli interventi di scavo, delle necessarie operazioni di manutenzione ordinaria e straordinaria, per la realizzazione di un programma di "attenzione" costante allo stato del sito finalizzato alla corretta conservazione dello stesso.

\section{IL SITO DI MASADA}

La caratteristica essenziale delle indagini che si occupano di grandi aree e complessi archeologici, come il sito di Masada inteso nel suo complesso territoriale, è quella di occuparsi di tutti i periodi testimoniati dalla stratificazione archeologica: nessun tipo di monumento, nessuna attività e nessun periodo risulta prioritario, poiché l'indagine conoscitiva che si prevede di mettere in atto parte, in primo luogo, dalla conoscenza del territorio in tutte le sue forme ed i suoi aspetti.

Il sito di Masada, individuato da un viaggiatore nel 1828 sugli aspri rilievi che si innalzano ad Est del Mar Morto, nella Giudea sud-orientale, si trova attualmente in territorio israeliano a circa cento chilometri a sudest di Gerusalemme. Il sito fu oggetto di studi da parte del grande specialista Schülte nel $1933^{11}$ e la grande fortezza venne meglio identificata e studiata dopo i primi scavi realizzati dal 1963 al 1965 dalla missione guidata dall'archeologo israeliano Yigael Yadin. ${ }^{12}$ Dal 1966 Masada ed il suo territorio divennero un'area protetta dal Ministero delle Antichità e dal 1998 fu dichiarata National Parks, Nature Reserves, National Sites and Memorial Sites. Divenuta sito protetto dall'UNESCO nel 2000 oggi è un

dell'analisi urbana e territoriale. La loro utilizzazione sta espandendosi fino a connotarli come un necessario strumento per l'organizzazione delle informazioni nel settore specifico del rilievo e della gestione dei dati per la pianificazione della conservazione dei siti di maggior interesse anche a livello territoriale.

11 A. Schülte, «Zeitschrift das deutschen Palästina - Vereins», LVI, 1933; M. Avi - Yonah, N. Avigad, Y. Aharoni, L. Dunayevsky, S. Gutman, The Archaeological Survey of Msada, 1955 - 56, «Israel Exploration Journal»,VII, 1957; Y. Yadin, The excavation of Masada 1963 - 1964. Preliminary Report, «Israel Exploration Journal», XV, 1965; Y. Yadin, Masada. Herod's Fortress and the Zelot's last Stand, London, 1966.

Macheronte: Győző Vörös, Machaerus I. History, Archaeology and Architecture of the Fortified Herodian Royal Palace and City overlooking the Dead Sea in Transjordan, Edizioni Terra Santa, Milano 2013. 
grande parco archeologico, uno dei più visitati in Israele, dotato di un ampio Visitors Center e di una funivia per il rapido collegamento con il sito principale dell'area, la fortezza, che occupa la porzione a nord della sommità della montagna. Una grandiosa cisterna artificiale sotterranea, assieme a numerosi altri invasi per la conservazione dell'acqua, sia sulla sommità del sito sia sviluppati sulle scoscese pendici del rilievo, dimostrano la lunga frequentazione antropica del sito che, come è attestato anche dagli storici, in particolare da Giuseppe Flavio, venne usato come fortificazione fino dal secondo secolo a. C. essendo costituito da un rilievo roccioso isolato dotato di due sole vie di accesso. ${ }^{13}$ Sulla sommità del rilievo, ad una altezza di circa quattrocento metri al di sopra della depressione del Mar Morto, si estende un pianoro della superficie di circa una decina di ettari (Fig. 1). Questo pianoro sommitale appare recinto da una singolare cortina muraria che sviluppa circa 1.300 metri, costituita da un doppio muro, composto dalla cortina esterna con torri di rinforzo e da un muro interno, collegati da murature trasversali disposte in maniera tale da formare una serie di vani comunicanti (sitema definito casamatta) una volta destinati a depositi, arsenali ed anche ad abitazioni (Fig. 2). Fra questi vani si distingue anche una sinagoga, ed alcune costruzioni destinate a colombari. All'interno del recinto fortificato appare ben evidente, nella zona Nord, un articolato complesso di costruzioni: il sistema dei magazzini, costituiti da due serie di fabbricati formati da lunghi vani (profondità variabile dai 20 ai 27 metri) modulati secondo una semplice viabilità interna, e grandi residenze disposte attorno a cortili, fra le quali emerge il cosiddetto Palazzo di Erode, risalente al I secolo a.C., un singolare complesso monumentale distribuito su tre terrazze del banco roccioso, sospese sul deserto con una incomparabile veduta del Mar Morto. ${ }^{14}$

12 Yigael Yadin con William F. Albright, fu uno dei principali rappresentanti di quella che viene chiamata "Archeologia biblica". Condusse gli scavi di Nahal Hever, sulle sponde del mar Morto e assurse a notorietà internazionale dirigendo lo scavo di Masada tra il 1963 e il 1965.

13 La storia di Masada è conosciuta principalmente dal lavoro di Giuseppe Flavio, lo storico ebreo del primo secolo d.C. Per la descrizione della Fortezza e degli apprastamenti di assedio dei romani cfr. Giseppe Flavio, La guerra giudaica, a cura di G. Vitucci, Arnoldo Mondadori Editore, ristampa 2012, cap. VII, 8, pp. 484-488.

$14 \mathrm{La}$ fase principale più importante nuova costruzione del Palazzo Nord è datata alla metà anni '20 del $1^{\circ}$ secolo a.C. Questo gruppo di edifici si trovano nel punto più alto della collina e costituiscono una acropoli difendibile della cittadella. 
All'interno dell'area di Palazzo vi è anche un edificio termale articolato in vari ambienti (oggi in buona parte restaurati) aperti su di una corte, con piscina e cisterne. Un ulteriore complesso palaziale con varie residenze occupa la parte sud occidentale del recinto fortificato ed appare composto da vari ambienti con singolari lunghi atrii di ingresso. ${ }^{15}$ Nella parte centrale dell'area vi sono i resti di un edificio di epoca bizantina, con una chiesa a pianta centrale, che testimonia l'occupazione del sito perlomeno fino a tale periodo. ${ }^{16}$ Molto interessanti e sufficientemente ben conservati appaiono anche i resti del grandioso apparato di assedio realizzato dai romani fra il 72 ed il $73 \mathrm{~d}$. C. costituito da un muro (circonvallazione) che circonda tutto il rilievo di Masada, rafforzato dalla presenza di ben otto campi militari fortificati con il tradizionale impianto quadrilatero. Fra le opere di assedio il resto più impressionante è costituito dalla rampa artificiale, realizzata in terra ed armata con strutture lignee (delle quali ancora rimangono tracce), che funzionava da piano inclinato per raggiungere le mura sulla cima del rilievo roccioso con una gigantesca torre d'assedio, resa famosa dagli scritti di Giuseppe Flavio. Lo straordinario stato di conservazione dell'intero complesso costituito dalla fortezza e dal territorio circostante con i resti delle opere di assedio ha fatto sì che tutta l'area venisse ritenuta di grande interesse e venisse dichiarata Patrimonio dell'umanità dall'UNESCO. ${ }^{17}$

15 Per la prima fase (c 35 a.C.) appartengono al nucleo del Palazzo occidentale tre palazzine, un edificio amministrativo, una caserma, tre colombari (utilizzati anche come torri di guardia), diversi grandi cisterne, e una piscina.

16 All'inizio della rivolta giudaica un gruppo di zeloti guidati da Menahem, occupano la fortezza di Masada, e molti ebrei si stabilirono lì dopo la caduta di Gerusalemme e la distruzione del Tempio. Flavio Silva, governatore romano, decise di eliminare questo ultimo centro residuo di resistenza ebraica ordinandone la conquista; su un sito roccioso sul versante occidentale di Masada fu costruita una rampa gigantesca per l'assedio delle mura. Cfr, cfr. Giseppe Flavio, La guerra giudaica, cit., Cap. VII, 8, p. 488.

17 UNESCO Declaration of Authenticity (2010): "This is a site that remained untouched for more than thirteen centuries. The buildings and other evidence of human settlement gradually collapsed and were covered over until they were revealed in the 1960s. There have been no additions or reconstruction, beyond an acceptable level of anastylosis, and inappropriate materials used in early conservation projects are being replaced. Limited restoration works have been carried out to aid visitor interpretation with original archaeological levels being clearly defined by a prominent black line set in the new mortar joints. Certain significant archaeological elements, such as the Roman camps and siegeworks, remain virtually untouched. The authenticity is therefore of a very high level". 


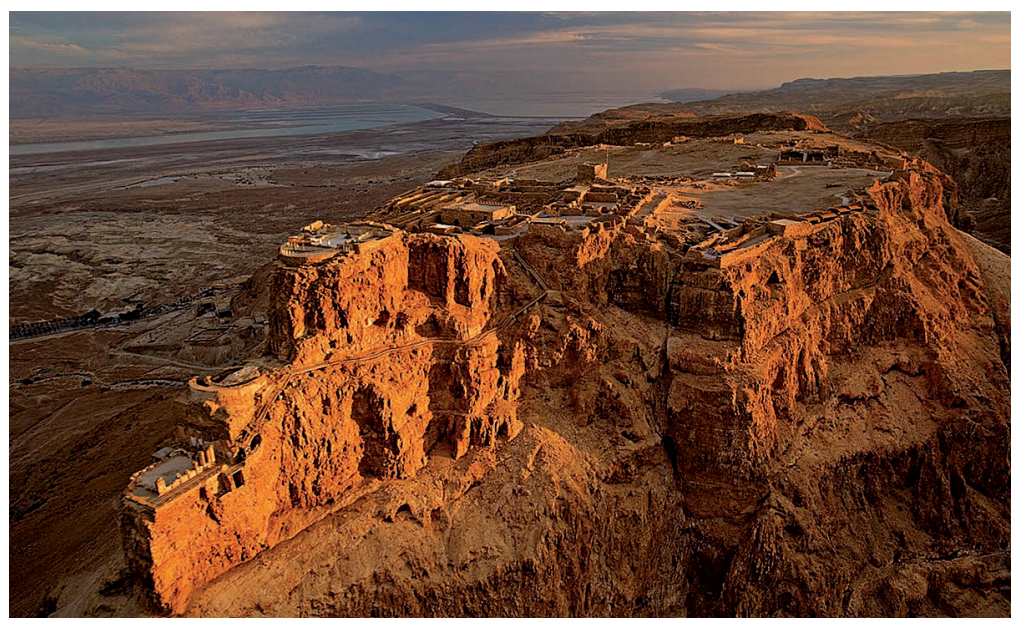

Fig. 1 - Foto aerea di Masada da Nord nella quale si apprezza la relazione tra il sito archeologico e la depressione del Mar Morto.

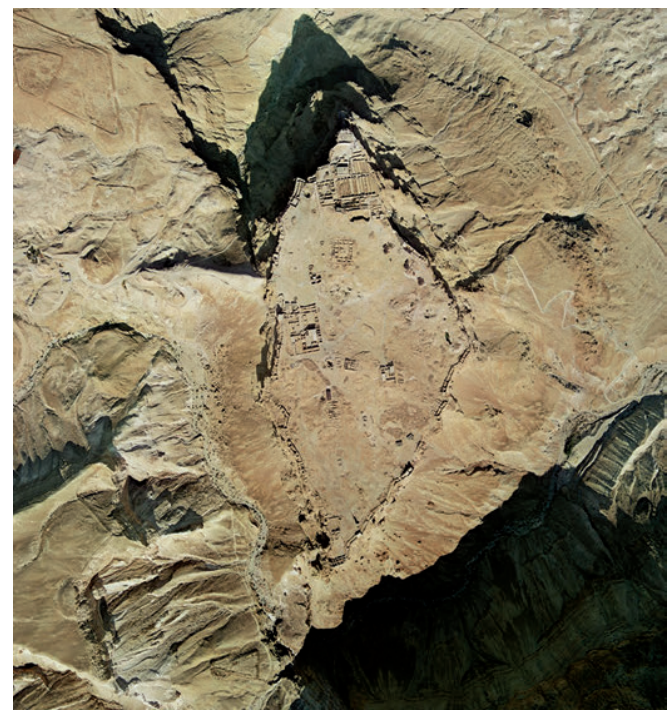

Fig. 2 - Ortofoto aerea del complesso di Masada.

L'esame del territorio, sia come ricerca preliminare per individuare la presenza di resti archeologici, sia per acquisire dati statistici generali sulla storia del territorio stesso, prevede di avvalersi in questo caso 
di un rilievo realizzato con scanner laser $3 \mathrm{D}$ che sarà integrato delle tradizionali metodologie di indagine (Figg. 3-4). ${ }^{18}$

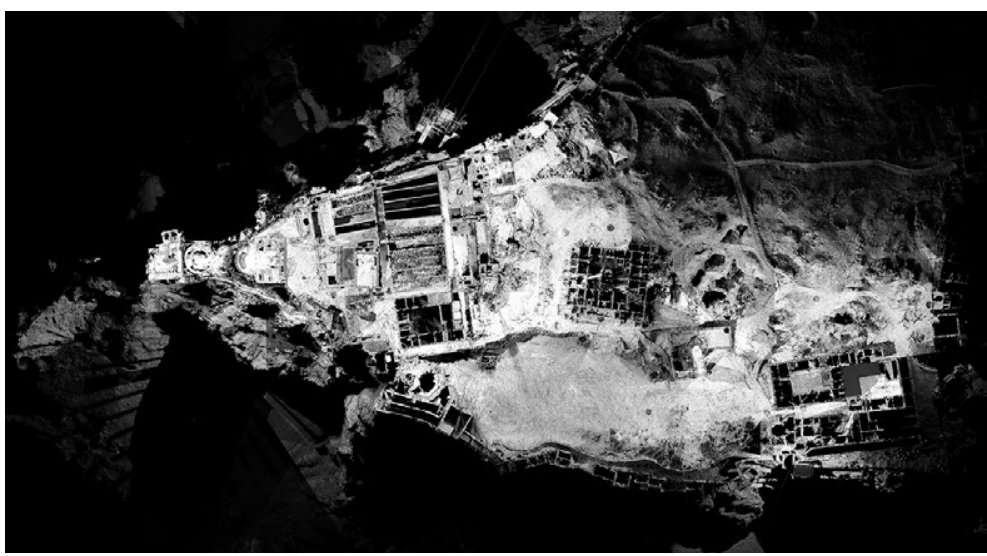

Fig. 3 - Banca Dati 3D - Nuvola di punti laser scanner (visualizzata in scala di grigio) relativa ai rilievi condotti per l'area Nord del plateau di Masada.

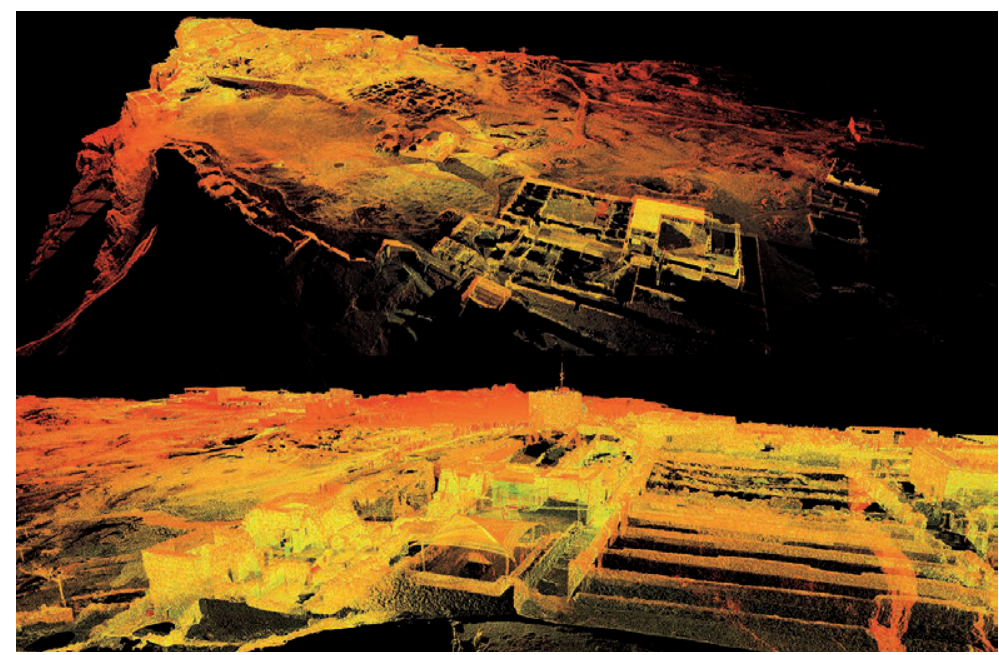

Fig. 4 - Vedute della nuvola di punti laser scanner.

18 Il progetto di rilevamento digitale, non esclude tuttavia la tradizionale ricognizione archeologica di superficie (osservazione diretta), l'interpretazione delle fotografie aeree ed eventuali prospezioni geofisiche. 


\section{UN PROGETTO PER LA DOCUMENTAZIONE DI MASADA}

Il programma di ricerca per la documentazione del sito archeologico di Masada prevede un ciclo di campagne di rilevamento organizzate in un triennio di attività estendibile in caso di sviluppo di progetti satellite che permettano l'incremento dei fondi a disposizione per le ricerche. Ciascuna missione è organizzata nella volontà di creare seminari tematici dove la ricerca si possa mescolare alla didattica e dove possano partecipare sia studenti, in genere degli ultimi anni dei corsi di laurea magistrale, che dottorandi di ricerca, ricercatori e docenti. Queste missioni, previste nei mesi di Febbraio, sono state organizzate in accordo tra $\mathrm{i}$ tre responsabili del progetto appartenenti alle tre Università capofila, Stefano Bertocci per l'Università di Firenze, Sandro Parrinello per l'Università di Pavia e Rebeka Vidal per l'istituto Shenkar di TelAviv, e si alternano ad altre missioni o workshop organizzate nei mesi di Settembre e Ottobre in Italia. Le attività italiane sono dedicate specificatamente alla formazione degli studenti tramite seminari tematici che permettano di approfondire lo studio delle tecnologie digitali nel campo della documentazione dei beni culturali mentre le attività condotte in Israele prevedono la messa in pratica di tali conoscenze operando su siti di interesse storico e archeologico.

Obiettivo generale delle ricerche in Israele è lo sviluppo e la sistematizzazione di una metodologia di acquisizione ed elaborazione dati relativi alle condizioni morfometriche del sito di Masada. Compresa dunque anche in forma apparente la complessità di un sistema così ampio, risulta necessaria un'organizzazione della struttura della ricerca inquadrando due aspetti principali: il primo riguardante gli obiettivi generali da un punto di vista tecnologico, relativi alle pratiche di rilevamento che si intendono sviluppare, il secondo che considera invece la dimensione del rilievo che, per un sito di così vaste dimensioni e così denso di elementi significanti, ricadrà su una pianificazione mirata delle attività.

La necessità di definire diversi ambiti specifici di interesse connessi all'immagine del luogo, corrisponde a quella necessità di inquadrare più informazioni possibili in grado di andare a ricostruire l'immagine complessa di questo sistema interconnesso di differenti realtà storiche, dal sito archeologico al moderno museo, nel quale ogni più modesto elemento si organizza secondo funzioni storiche che il rilievo deve poter sapere interpretare. 
La banca dati dei rilievi realizzati, utile strumento di controllo per lo sviluppo dell'intero sito archeologico permetterà, in fase di elaborazione, di giungere a disegni affidabili su quanto presente oggi all'interno del museo all'aperto, consentendo inoltre la digitalizzazione e la virtualizzazione di ogni singolo elemento architettonico (Figg. 5-6-7).
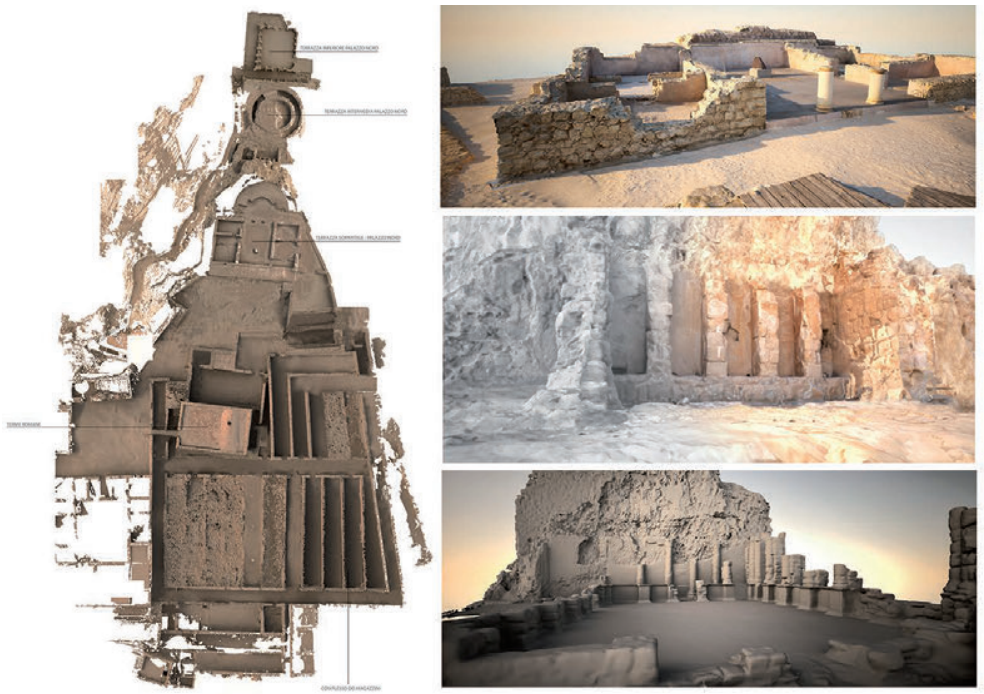

Fig. 5-Modellazione 3D mediante mesh poligonale, della nuvola di punti relativa al Palazzo Nord di Masada e caratterizzazione delle superfici mediante applicazione di fotopiano.

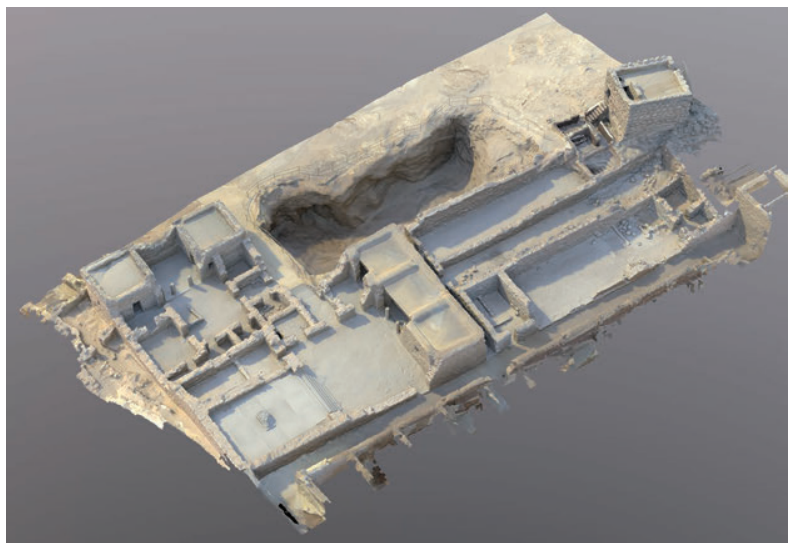

Fig. 6-Modellazione del palazzo del Capitano mediante fotogrammetria structure from motion. 

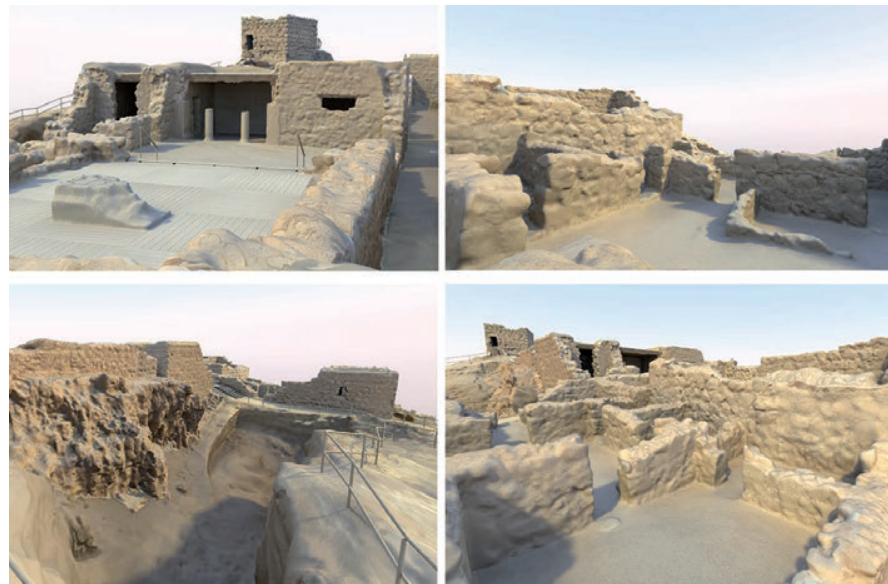

Fig. 7 - Visualizzazione del modello fotogrammetrico realizzato per l'area del palazzo del Capitano.

Questo fenomeno implica la possibilità di creare ulteriori piattaforme informative attraverso le quali operare una procedure di valorizzazione del sito stesso tramite web.

La possibilità di interagire virtualmente con l'architettura di Masada permette di sperimentare ciascuna scelta progettuale e di intervenire con simulazioni anche in relazione a sviluppi di pianificazione (Fig. 8). I siti UNESCO oggi necessitano di attività come queste per determinare con maggior metodo e controllo le politiche di gestione e di sviluppo del sito.

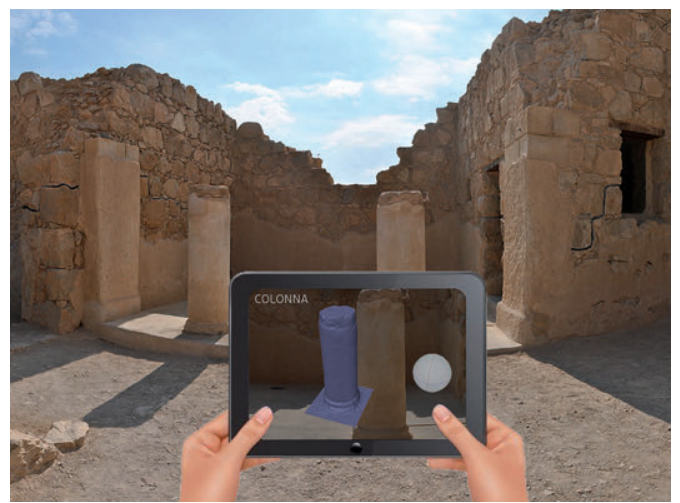

Fig. 8 - Interazione tra strumenti multimediali ed elementi costruttivi per lo sviluppo del sistema museale del complesso di Masada. 


\section{RIFERIMENTI BIBLIOGRAFICI}

Stefano Bertocci, Sandro Parrinello. Digital Survey and Documentation of the Archaeological and Architectural Sities. Unesco World Heritage List. p. 1-240, Firenze:Edifir-Edizioni Firenze, 2015.

Stefano Bertocci, Sandro Parrinello, Rebeka Vital (a cura di), MASADA NOTEBOOKS REPORT OF THE RESEARCH PROJECT 2014. vol. II, Firenze:Edifir-Edizioni Firenze, 2014.

Stefano Bertocci, Sandro Parrinello, Rebeka Vital (a cura di). MASADA NOTEBOOKS REPORT OF THE RESEARCH PROJECT 2013. vol. I, Firenze:Edifir-Edizioni Firenze, 2013.

Bertocci Stefano, Bini Marco. Manuale di rilievo architettonico e urbano Torino, Città Studi Edizioni, De Agostini Scuola, 2012.

Sandro Parrinello, Francesca Picchio, Dalla fotografia digitale al modello 3D dell'architettura storica. DISEGNARE CON..., vol. 6, p. 1-14, ISSN: 1828-5961, doi: 10.6092/issn.1828-5961/3870, 2013. 